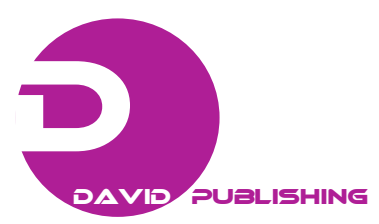

\title{
Deep Semantic Structure of Natural Language
}

\author{
Nabil Hassan \\ Electrical Engineering Department, William Vacanarat Shadrach Tubman College, Harper, Maryland county Liberiare
}

\begin{abstract}
This paper presents the result of research of deep structure of natural language. The main result attained is the existence of a deterministic mathematical model that relates phonetics to associated mental images starting from the simplest linguistic units in agreement with the human response to different acoustic stimuli. Moreover, there exists two level hierarchy for natural language understanding. The first level uncovers the conceptual meaning of linguistic units, and hence forming a corresponding mental image. At the second level the operational meaning is found to suit, context, pragmatics, and world knowledge. This agrees with our knowledge about human cognition. The resulting model is parallel, hierarchical but still concise to explain the speed of natural language understanding.
\end{abstract}

Key words: Natural language model, conceptual meaning, operational meaning, composition.

\section{Introduction}

Natural languages are our basic medium for communication and logical thinking. However, they are ambiguous and their mechanisms are poorly understood. Typical examples are illustrated in example 1 and example 2. This research is the first to show that natural languages have hierarchical deep structure that binds naturally phonetics to mental images. In higher levels of processing the operational meaning is determined by considering context, pragmatics, and world knowledge.

Example 1 The semantics of the message "ninety percent" is obvious. However, the phrase meaning differs for someone expecting results of examination and another expecting stock market price of his shares. Such ambiguity is solved via information theory and pragmatics.

Example 2 The fragment "During heading to receive my brother arriving from abroad at eight $\mathrm{I}$ smelled smoke that required fixing" does not mention the words "airport", "plane", "hour", "car", "workshop", but these are inferred from world knowledge.

Corresponding author: Nabil Hassan, Ph.D., professor and chair, research fields: systems, AI. E-mail: profdrnabi163@yahoo.com.
Both examples illustrate that natural language understanding is a complex process that includes meaning of linguistic units, context, pragmatics, world knowledge and interaction between author and reader. Fig. 1 depicts a model for natural language understanding.

The resulting model was tested against all words in the Arabic lexicon (precisely the intermediate lexicon that proves the completeness of the model. This tedious procedure extends for seven years plus.

The paper shows the mainly the first level of the hierarch; i.e., the relation between phonetics and conceptual meaning of letters, then proceeds to composition of letters into words.

The following sections of the paper present the model in a stepwise procedure. Section 2 introduces the basic structure of the model. Section 3 depicts the evolution of alphabet letters from the bases model. Section 4 shows how to compose letters into words and illustrates the procedure with two examples. Then come conclusions, and Appendix summarizes the meaning of letters of alphabet, used symbols together with useful categorization of letters.

\section{Theory for Alphabet Letters Semantics}

The meaning of alphabet letters is based on three 


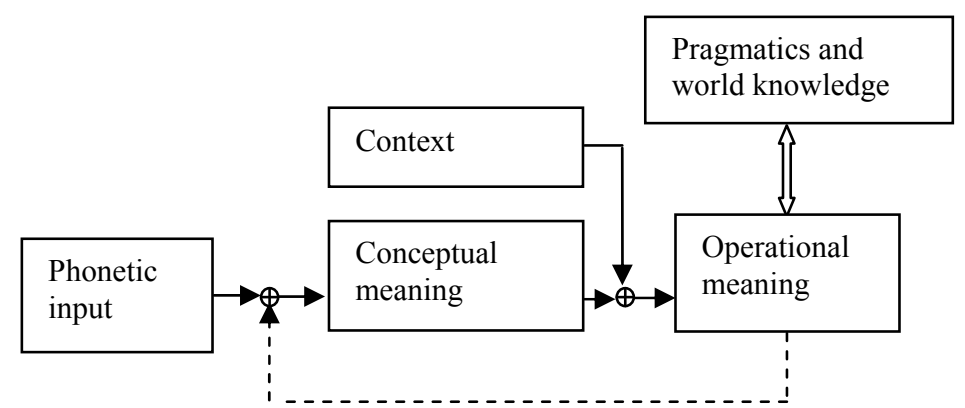

Fig. 1 Natural language understanding paradigm, the bottom up processing is complemented with possible top-down processing materialized by the dashed line.

sets: namely, the set of components, the set of relations, and the anonymous set, and could be colored via two primary attributes $\{\mathcal{A}, \mathcal{P}\}$, for activity/effectiveness, and passivity/ineffectiveness, and two secondary attributes $\{\mathfrak{R}, \mathfrak{I}\}$ for real and imaginary, $\mathfrak{R}$ refers also to tangible, or sensible; $\mathfrak{I}$ refers also to being transparent, hidden or conceptual. Frame F1 depicts composition of attributes via exclusive OR (XOR).

F1

(1) $\mathcal{P} \circ \mathcal{P}=\mathcal{A} \circ \mathcal{A}=\mathcal{A}$

(2) $\mathcal{P} \circ \mathcal{A}=\mathcal{A} \circ \mathcal{P}=\mathcal{P}$

(3) $\mathfrak{I} \circ \mathfrak{I}=\mathfrak{N} \circ \mathfrak{N}=\mathfrak{N}$

(4) $\mathfrak{I} \circ \mathfrak{N}=\mathfrak{N} \circ \mathfrak{I}=\mathfrak{I}$

\subsection{Components Set $\mathbb{C}$}

The elements of components set $\mathbb{C}$ are source $(\mathcal{S})$ and medium $(\mathcal{M})$, and each could be attributed as depicted symbolically in table 1 , and hence they could be equally represented algebraically or graphically. The later provides more inspiration and permits the construction of semantic graphs.

The word source $\mathcal{S}$ is understood also as origin, provenance, antecedent, or exporter. Active real source is also tangible or sensible. An active imaginary source is hidden, conceptual, or motive. A passive real source is a sink. An imaginary sink is extremity, conceptual or absorber. A medium $\mathcal{M}$ is a barrier, separator, or isolator. An active real medium is tangible or sensible. Passive real medium is apex, cover, or verge. Active imaginary medium is transparent, conceptual, or penetrative. Passive imaginary medium is a hypothetical apex, limit or cover.

\subsection{Relations Set $\mathbb{R}$}

The elements of relation set $\mathbb{R}$ deportation and conjunction $\mathcal{C}$, and each could be colored with $\mathcal{A}, \mathcal{P}$, $\mathfrak{R}$ or $\mathfrak{I}$ as depicted symbolically in Table 2.

$\mathcal{D}$ refers also to expelling, displacing or dislodging. Passive deportation is attraction, dragging, or drawing. Active imaginary deportation refers to propagation, diffusion, scattering or partial deportation. Passive imaginary deportation indicates taking over, acceptance, acquiring, or attraction of part. Conjunction $\mathcal{C}$ is also understood as grouping in a whole, synchronization, or concurrency. Active real conjunction is tangible or sensible, and hence limited in duration, space or quality. Passive real conjunction is stuffing of unrelated objects in space, time, or quality without forming a whole. Active imaginary conjunction is permanent or regular. Passive imaginary conjunction is hypothetical, permanent (recursive), or conjunction of separated objects.

Table 1 Symbols for components.

\begin{tabular}{|l|l|l|l|l|}
\hline \multirow{2}{*}{} & \multicolumn{2}{|c|}{$\mathcal{A}$} & \multicolumn{2}{c|}{$\mathcal{P}$} \\
\cline { 2 - 5 } & $\Re$ & $\mathfrak{I}$ & $\Re$ & $\mathfrak{J}$ \\
\hline $\mathcal{S}$ & & & & \\
\hline $\mathcal{M}$ & & & & $\mathcal{Y}$ \\
\hline
\end{tabular}

Table 2 Symbols for relations.

\begin{tabular}{|l|l|l|l|l|}
\hline \multirow{2}{*}{} & \multicolumn{2}{|c|}{$\mathcal{A}$} & \multicolumn{2}{c|}{$\mathcal{P}$} \\
\cline { 2 - 5 } & $\Re$ & $\mathfrak{I}$ & $\Re$ & $\mathfrak{I}$ \\
\hline $\mathcal{D}$ & $\longrightarrow$ & $-\rightarrow--$ & $\longrightarrow$ & ---- \\
\hline $\mathcal{C}$ & $\longleftrightarrow$ & $\longleftrightarrow-\rightarrow$ & $\longleftrightarrow$ & $\longleftrightarrow--$ \\
\hline
\end{tabular}




\subsection{Anonymity Set MI}

Anonymous elements, depicted symbolically in Table 3, represent unknown components that arise commonly in natural language communication, and hence complete the system.

When a source $\mathcal{S}_{1}$ composes with anonymous source $\mathcal{S}$, the former inherits attributes from the later, in analogy with the relations between nouns and pronouns as shown in frame $\mathrm{F} 2$.

F2

$$
\begin{aligned}
& \mathcal{S}_{1} \circ \mathfrak{R S}=\mathcal{S}_{1}, \mathcal{S}_{1} \circ \mathfrak{J} \mathcal{S}=\mathfrak{I} \mathcal{S}_{1}, \\
& \mathcal{S}_{1} \circ \mathcal{P} \mathcal{S}=\mathcal{P} \mathcal{S}_{1}, \mathcal{S}_{1} \circ \mathcal{P} \mathfrak{J} \mathcal{S}=\mathcal{P} \mathfrak{J} \mathcal{S}_{1}
\end{aligned}
$$

\section{Arabic Alphabetic Letters}

Arabic alphabet comprises consonants, short vowels, and long vowels. Arabic consonants are used exclusively with short vowels acting as identifiers,

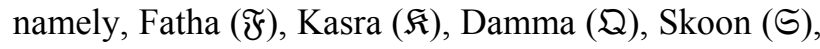
Madda (M) and Shadda $(\mathfrak{X})$. The first three short vowels are the composition of imaginary attribute with corresponding vowel, i.e., $\mathfrak{F}=\mathfrak{J} \circ \operatorname{Aleph},(') \mathfrak{K}=$ $\mathfrak{J} \circ$ Yae (ي), $\mathfrak{D}=$ J० Waw (و). $\mathfrak{M}=\mathrm{L} \circ \mathrm{L}, \mathrm{L}$ is an arbitrary alphabet letter represent recursion of $\mathrm{L}$; mostly Aleph. $\mathfrak{X}$ results in forced association in permanent recursion or conjunction of separated entities, and this normally requires instigator agent. $\mathbb{S}$ is a separated stuffing.

There are two types of letters: single element letters (one-tuple) and three elements letters (three-tuple), representing two components and the relations between them as depicted in Fig. 2. Short vowels colors both types; $\mathfrak{F}$ adds the attribute primary, $\mathfrak{K}$ adds secondary, $\mathfrak{D}$ adds permanent or regular, and $\subseteq$ adds separated stuffing. In three-tuple letters short vowels add selection of a particular component. $\mathfrak{F}, \mathcal{D}$ select the right component in the pair, $\mathfrak{K}$ chooses the left component, and $\widetilde{S}$ chooses the couple together. Tables 4 and 5 depict the detailed symbols of letters. The rows of the first column in each are representative of a basic quaternary system. Later columns classify letters into four classes according to the primary and secondary attributes.

\section{Composing Letters into Words}

The first thing to consider is rules to compose the constituent elements, and hence rules of composition.

\subsection{Composing Relations}

F3

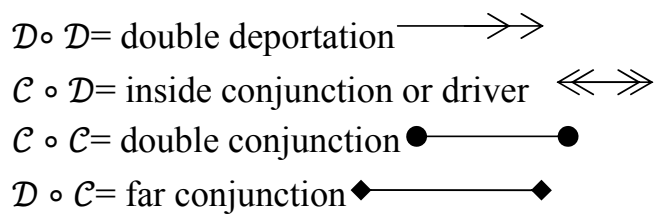

The resulting attributes are inferred from the constituent attributes by rules in frame F1.

\subsection{Composing Sources}

Sources compose with anonymous components according to the rules of frame F1. They may also compose with other sources or media forming a couple. That is,

Table 3 Symbols for anonymity.

\begin{tabular}{|l|l|l|l|l|}
\hline \multirow{2}{*}{} & \multicolumn{3}{|c|}{$\mathcal{A}$} & \multicolumn{2}{c|}{$\mathcal{P}$} \\
\cline { 2 - 5 } & $\Re$ & $\mathfrak{J}$ & $\Re$ & $\mathfrak{J}$ \\
\hline $\mathbb{M}$ & & $\square$ & & $\square$ \\
\hline
\end{tabular}

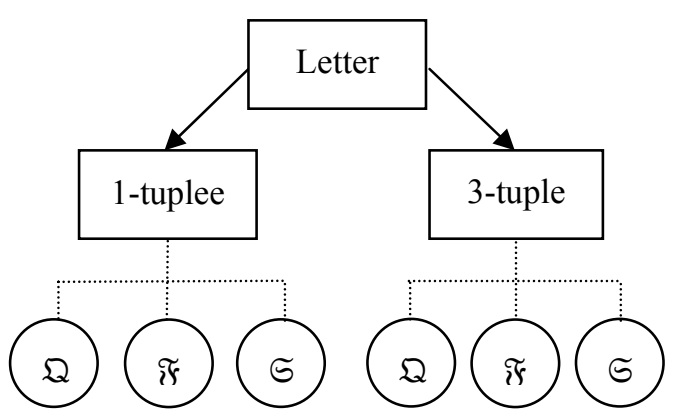

Fig. 2 Classification of letters with possible attributes.

\begin{tabular}{|c|c|c|c|c|}
\hline & & $\mathcal{A}$ & & $\mathcal{P}$ \\
\hline & $\Re$ & $\mathfrak{I}$ & $\Re$ & $\mathfrak{J}$ \\
\hline $\mathcal{S}$ & & $D$ & & $\varnothing$ \\
\hline $\mathcal{M}$ & ف & ${ }_{2} \mathrm{O}$ & & $D$ \\
\hline c & $\leftrightarrow$, & 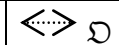 & S & $\mathfrak{X} \leftrightarrow$ \\
\hline $\mathcal{D}$ & $1>-$ & 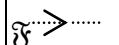 & $\leftrightarrow \longrightarrow$ & $\mathfrak{K} \cdots \cdots$ \\
\hline
\end{tabular}

Table 4 one -tuple letters. 
Table 5 three -tuple letters.

\begin{tabular}{|c|c|c|c|c|}
\hline & & $\mathcal{A} \mathbb{R}$ & & $P \mathbb{R}$ \\
\hline & $\Re$ & $\mathfrak{I}$ & $\mathfrak{R}$ & $\mathfrak{I}$ \\
\hline & & & $ث$ & \\
\hline $\mathcal{A S}$ & ن & 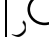 & ذا & ز \\
\hline $\mathcal{P S}$ & $\dot{ }$ & ج & $\tau$ & u \\
\hline $\mathcal{A M}$ & & & ق & \\
\hline $\mathcal{P} \mathcal{M}$ & ظ & ش & $b$ & $J$ \\
\hline
\end{tabular}

$\mathcal{S} \circ \mathcal{S}=$ two coupled sources

$\mathcal{S} \circ \mathcal{M}=$ outside conjunction of a source by a medium

$\mathcal{M} \circ \mathcal{M}=$ two coupled media

$\mathcal{M} \circ \mathcal{S}=$ inside conjunction of a source with a medium

The type of coupling is determined by the relation between two letters. The resulting attributes are determined by rules in frame F1.

\subsection{Procedure 1: Joining Letters into Words}

(1) Project letters of the word using symbols in Table A1 and/or codes in Tables 4 and 5;

(2) Add the coloring attributes dictated by short vowels;

(3) Compose the relations and attributes and simplify the result;

(4) The long vowels dissects the word into independent sections joined by the long vowel;

(5) The short vowel associated with the last letter determines the relation to logically next word. Logically stands for meaning in contradiction to lexical order.

"كوب" Example 3 The word

The meaning of this word in English is "cup". We follow procedure 1 as follows:

(1) The letters included together with their graphical representation is

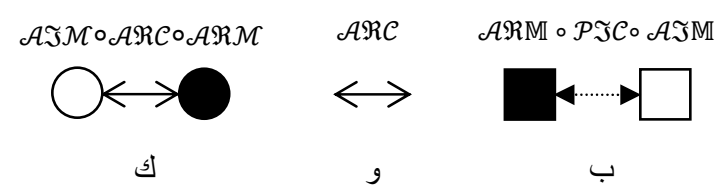

(2) We add the short vowels to get

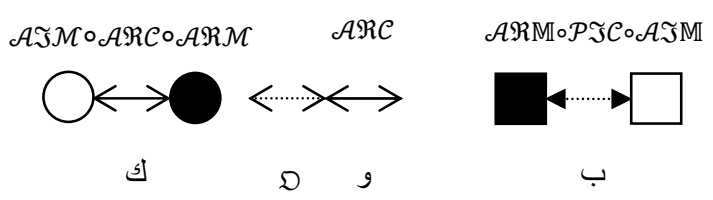

(3) Compose relations to get

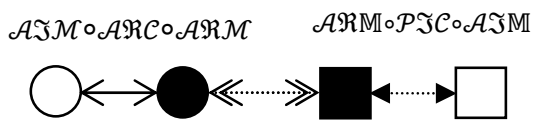

$\mathcal{A} \Im \mathcal{C} \circ \mathcal{A} \Im \mathcal{C}$

(4) Compose the anonymous and move the interrelation to the interrelation to get

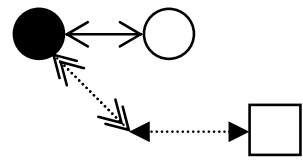

(5) Further compose the relation

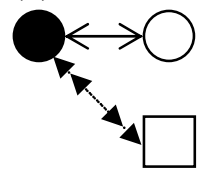

The conceptual meaning becomes: A real medium conjunct with an imaginary medium and could be conjunct internally recursively with anonymous imaginary object. The analysis of the English word "cup" gives similar conceptual meaning. I examined plenty of words in this way and obtained the same results, however, the proof of general validity needs more experiments.

"وازع" Example 4 The word

The corresponding meaning in English is "self authority for approval or disapproval". This could be developed based on faith or practice. Application of procedure 1 follows.

(1) We list the letters in the word.

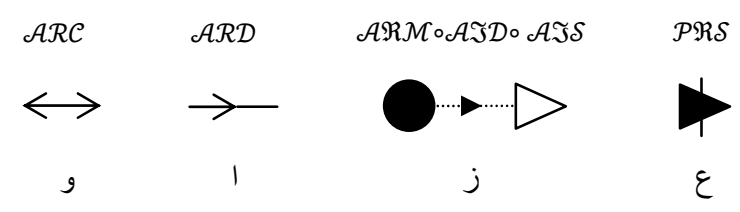

(2) Add short vowels while noting that $\mathfrak{K}$ selects the left member in three-tuple letters and makes it secondary. 
(3)

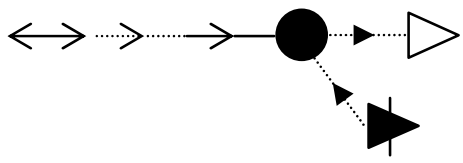

(4) Compose the two $\mathcal{D}$ relations taking care about the attributes.

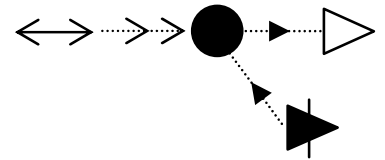

(5) Compose $\mathcal{C}$ and $\mathcal{D}$ where the symbol denotes imaginary internal conjunction (frame F3)

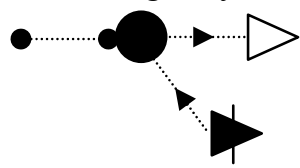

This result agrees with the mental image of this word. It is an internal motive associated with an object that accepts form imaginary source or real sink. Analyzing the English word "wise" that have similar pronunciation to our word gives a similar result.

\section{Conclusions}

The research gives a complete model for the deep structure of Arabic language. However, there are important cues that other languages have the same systemic features and insight on otherwise unknown phenomena associated with natural languages. For example:

$\odot \quad$ the speed of human processing and communication via natural languages;

$\odot$ the textual semantic ambiguity of natural languages;

$\odot$ why new learners, including children learning their mother language, acquire the language by just learning few words, estimated as two hundred, without smallest knowledge of grammar;

$\odot$ the relation between intelligence and the elegancy of implemented language;

$\odot$ the systemic nature of natural language that dictates a set of few words for arbitrary object. This set is not completely arbitrarily but must keep precise relations to the whole vocabulary;

$\odot$ language is dynamic; mainly vocabulary evolves with the evolution of knowledge;

$\odot$ the social effect on natural languages.

Future work should include the representation of letters with phonetic symbols to study the general applicability of this model, and the study of the relation between context and operational meaning.

Appendix

Table A1 Denotation of Arabic letters.

\begin{tabular}{|c|c|c|c|c|c|}
\hline$\leftrightarrow \leftrightarrow 0$ & $\begin{array}{l}\text { ق } \\
21 \\
\end{array}$ & $a \rightarrow-\Delta$ & \begin{tabular}{|l} 
\\
11 \\
\end{tabular} & $>-$ & $\begin{array}{l}1 \\
1 \\
\end{array}$ \\
\hline $0<$ & $\begin{array}{l}5 \\
22 \\
\end{array}$ & $0<$ & $\begin{array}{l}\omega \\
12 \\
\end{array}$ & $\square$ & \begin{tabular}{|l} 
\\
\end{tabular} \\
\hline$\alpha-\phi$ & $\begin{array}{l}J \\
23\end{array}$ & 0 & $\begin{array}{l} \\
13 \\
\end{array}$ & & $\begin{array}{l}ت \\
3\end{array}$ \\
\hline 0 & $\begin{array}{l}\text { ? } \\
24 \\
\end{array}$ & & ص & & $\begin{array}{l} \\
4 \\
\end{array}$ \\
\hline$O \rightarrow$ & $\begin{array}{l} \\
25 \\
\end{array}$ & $\phi$ & ض & & $\begin{array}{l} \\
5\end{array}$ \\
\hline$D$ & \begin{tabular}{|l|} 
\\
26 \\
\end{tabular} & $\phi$ & $\begin{array}{l} \\
16 \\
\end{array}$ & $\rightarrow \phi$ & $\begin{array}{l}2 \\
6 \\
\end{array}$ \\
\hline$\longleftrightarrow$ & $\begin{array}{l}9 \\
27 \\
\end{array}$ & O & $\begin{array}{l}b \\
17 \\
\end{array}$ & $O \rightarrow-1$ & $\begin{array}{l}\dot{z} \\
7 \\
\end{array}$ \\
\hline$\rightarrow$ & $\begin{array}{l} \\
28\end{array}$ & 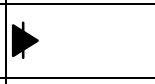 & $\begin{array}{l}\varepsilon \\
18 \\
\end{array}$ & $>0$ & $\begin{array}{l}2 \\
8\end{array}$ \\
\hline$D$ & 29 & 1 & \begin{tabular}{|l|}
$\dot{\varepsilon}$ \\
19 \\
\end{tabular} & $\rightarrow$ & $\begin{array}{l}\dot{3} \\
9 \\
\end{array}$ \\
\hline 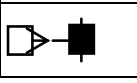 & $\begin{array}{l} \\
30 \\
\end{array}$ & 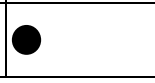 & $\begin{array}{c} \\
20 \\
\end{array}$ & $\mathrm{O} \rightarrow-\rightarrow$ & $\begin{array}{l} \\
10 \\
\end{array}$ \\
\hline
\end{tabular}




\begin{tabular}{|l|l|l|l|l|l|}
\hline$-\rightarrow--$ & $\mathfrak{F}$ & $\leftrightarrow \rightarrow$ & $\mathfrak{S}$ & \multicolumn{1}{|c}{} \\
\hline$\phi$ & 33 & 32 & 31 \\
\hline
\end{tabular}

We can further abstract the meaning of the letters in Table A1. To this end we categorize the letters into the following groups:

(1) Letters acting as sources: 26, and 29. Letter 26 is a stimulator, and letter 29 is a prevailing source.

(2) Letters acting like medium: 20 and 24. Letter 20 is a non-transparent barrier, and letter 24 is a transparent medium.

(3) Letters focusing on relations between intervening objects, namely 1, 2, 3, 4 and 28. The letters 1 and 28 represents deportation and attraction, respectively. Letter 2 represents neighborhood, letter 3 represents diffusion, and letter 4 represents partially separated conjunction.

(4) Letters focusing on conjunction: 20,21, 22, and 27. The letter 27 represents grouping in a whole, synchronization, or concurrency, letter 21 is partial conjunction of objects of different quality.

(5) Letters focusing on support: 12,13,18, and 19. Letter 12 is a real sink, letter 13 is a cover, letter 18 is regular support of a real object to imaginary object, and letter 19 is a regular support of a cover to imaginary medium.

(6) Letters focusing on drift or tendency: 14, 15, 16, 17, and 23. Letter 14 is spread or propagation towards an extremity, letter 15 is anonymous extremity, letter 16 is separated conjunction of a hypothetical apex with a real medium.

(7) Letters focusing on remote connection: 5, 6, 7, and 25. Letter 5 represents the spread of a real sink from a transparent medium, letter 6 represents a separated conjunction of a boarder from a nontransparent medium, letter 7 represents the attraction, dragging, or draw of imaginary medium to real sink, and letter 25 represents refers to expelling or dislodging of a prevailing source from an imaginary medium.

(8) Letters focusing on coupling/association/union: 8, 9, 10,11, and 12. The letter 8 represents hypothetical permanent coupling between transparent medium and nontransparent medium, letter 9 represents passive coupling of a stimulator to nontransparent medium (physical object), letter 10 represents propagation/emission that couples prevailing source to transparent medium, and letter 12 represents permanent coupling between nontransparent (opaque) object and a transparent object.

Abstraction at the level of letter, helps forming words then sentences.

Table A2 Used symbols.

\begin{tabular}{|l|l|l|l|}
\hline Bases sets & Primary attributes & Components & Short vowels \\
\hline \multirow{3}{*}{$\begin{array}{l}\text { C set of components } \\
\mathbb{R} \text { set of relations }\end{array}$} & $\mathcal{A}$ activity/positivity & $\mathcal{S}$ source $\mathcal{M}$ medium & $\begin{array}{l}\mathfrak{f} \text { fatha } \\
\mathfrak{K} \text { kasra } \\
\mathfrak{S} \text { skoon }\end{array}$ \\
\cline { 2 - 4 } & Secondary attributes & Relations & Doubling \\
\cline { 2 - 4 } & $\begin{array}{l}\mathfrak{R} \text { tangible/sensible } \\
\mathfrak{I} \text { imaginative }\end{array}$ & $\begin{array}{l}\mathcal{D} \text { deportation } \\
\mathcal{C} \text { conjunction }\end{array}$ & $\begin{array}{l}\mathfrak{D} \text { Damma } \\
\mathfrak{X} \text { shadda } \\
\mathfrak{M} \text { madda }\end{array}$ \\
\hline
\end{tabular}

